\title{
Los moluscos terrestres (Mollusca: Gastropoda) de Costa Rica: clasificación, distribución y conservación
}

\author{
Zaidett Barrientos Llosa \\ Laboratorio de Ecología Urbana, Universidad Estatal a Distancia, 474-2050 San Pedro de Montes de Oca, San José, \\ Costa Rica; zbarrientos@uned.ac.cr
}

Recibido 26-VII-2010. Corregido 01-VIII-2010. Aceptado 04-VIII-2010.

\begin{abstract}
Terrestrial mollusks (Mollusca: Gastropoda) of Costa Rica: classification, distribution and conservation. Terrestrial mollusks are poorly known worldwide. The country has 183 reported species, 30\% endemic and 7\% are probably extinct. About 400 species are expected to inhabit the country. Biology, ecology, distribution, genetics and other areas of research are unknown for more than $95 \%$ of the species. The most diverse families are Spiraxidae, Orthalicidae and Subulinidae. However, the family that may have more species is Euconulidae. Euconulids inhabit the highlands, where less work has been done. The study of species of highlands will also rise the endemism rate. Future taxonomic, biological and ecological work should consider their low vagility, tendency to produce new taxa in sympatry, specific microhabitat requirements, hermaphroditism, high evolutionary rate ( $10 \%$ per million years), and divergence between species ( 2 to $30 \%$ ). Urgent studies to protect the Costa Rican malacofauna include: distribution, abundance, effect of land use and climate changes on populations. Rev. Biol. Trop. 58 (4): 1165-1175. Epub 2010 December 01.
\end{abstract}

Key words: land snails, endemism, invertebrate conservation.

Los moluscos son un grupo de invertebrados muy diverso, sólo superado por los artrópodos (Margulis \& Schwartz 1998). La mayor parte de los moluscos vive en el mar, pero también existen especies dulceacuícolas y terrestres. Las especies terrestres son pequeñas, poco coloridas, difíciles de encontrar y difíciles de estudiar, por lo tanto, existen muchos vacíos en el conocimiento del grupo (Monge-Nájera 1997). Presentan, además, una gran cantidad de endemismos y se calcula que alrededor de un $50 \%$ de las especies no han sido descritas. El porcentaje de las cuales se conoce algún aspecto de su biología o ecología es mucho menor (Pérez et al. 2008).

Los moluscos terrestres son importantes para los humanos porque: transmiten enfermedades tanto a las personas como a los animales domésticos; se utilizan como alimento y en la fabricación de artesanías; se les emplea como especies bioindicadoras de contaminación (Dallinger et al. 2001, Achuba 2008); forman parte del reciclaje de nutrientes en los bosques (Dallinger et al. 2001); son fuente de alimento para aves, culebras, ratones e invertebrados (Sazima 1989); y son plagas agrícolas (Jennings \& Barkham 1979, Monge-Nájera 1997). Además, a nivel paleoclimático se ha encontrado que las comunidades de moluscos terrestres son buenos bioindicadores de temperatura, precipitación y biodiversidad en general (Rousseau 1992).

En Costa Rica se ha informado la presencia de 183 especies, pero solo se conoce parte de la ecología y biología de aproximadamente cinco, de las cuales dos son especies introducidas y las restantes tienen importancia agrícola (Andrews et al. 1985, Villalobos et al. 1995, Barrientos 1998, 2000). 


\section{CLASIFICACIÓN}

\section{Taxonomía}

Se calcula que en el mundo hay alrededor de 24000 especies descritas de moluscos terrestres y que faltan por describir entre 11 000 y 40000 (Lydeard et al. 2004). De Costa Rica se han registrado 183 especies de moluscos terrestres (Barrientos 2003a), no obstante, podría haber unas 400 especies (F.G. Thompson 2000 , com. pers.). La descripción de la mayoría de las especies se realizó en forma aislada -no dentro del contexto de un género-, incompleta (a menudo únicamente la concha) y solo de una localidad, sin prestar atención a la variabilidad de la especie. Además, debido a que falta describir muchas especies y a que se desconocen las variaciones morfológicas naturales de la mayoría, es necesario realizar una revisión completa de la mayor parte de los géneros presentes en el país (F.G. Thompson 2000, com. pers., Barrientos 2005).

En Costa Rica las familias con más cantidad de especies descritas son Spiraxidae (con 33spp.), Orthalicidae (30spp.) y Subulinidae (23spp.) (Barrientos 2005). La familia Euconulidae ocupa el quinto lugar con 14 especies informadas del país. No obstante, esta familia es más diversa en zonas de más de $1000 \mathrm{msnm}$ y la mayoría de las especies descritas hasta el momento habitan en zonas bajas, por lo tanto, es probable que en realidad la familia Euconulidae sea la más diversa en Costa Rica. Por ejemplo, se puede citar que solo en el Valle del Silencio, Cordillerra de Talamanca, se encontraron 14 especies aún no descritas de esta familia (Barrientos 2005). La familia Euconulidae, orden Stylommatophora, está distribuida en todo el mundo y consta de dos subfamilias (Schileyko 2002). La subfamilia Microcystinae está ausente en América y de la subfamilia Euconulinae solamente están presentes tres géneros de los 27 que la conforman: Habroconus Fisher \& Crosse, Velifera Binney y Euconulus Reinhardt (Schileyko 2002). Costa Rica es el único país en el que están presentes estos tres géneros, por lo que se facilita el estudio de las relaciones filogenéticas del grupo en el continente. El género Еисопиlus se divide en nueve subgéneros y cuatro de ellos tienen representantes en América: Guppya Moerch 1867, Pseudoguppya Baker 1925, Dryachloa Thompson \& Lee, 1980 -solo este subgénero no está presente en Costa Rica- y Euconulops Baker, 1928 (Schileyko 2002). El género Habroconus tiene cuatro subgéneros (Habroconus Fischer et Crosse, 1872, Cocosconus Baker, 1941, Ernstia Jousseaume 1889 y Cocoslens Baker, 1941), (Schileyko 2002) y es posible que Costa Rica sea el único país en donde se encuentren los cuatro subgéneros. Velifera es un género endémico de Costa Rica, a la fecha solo existe una especie descrita, Velifera gabbi Binney, 1879 (Binney 1879). Esta especie fue recolectada en los Cerros de Escazú, San José y los especímenes fueron depositados en el Museo de la Academia de Ciencias de Filadelfia, Estados Unidos, donde fueron vistos por última vez en 1997. Actualmente están perdidos, por lo tanto es posible que se deba designar un neotipo. La descripción original cuenta con un buen dibujo de la especie pero se desconocen su anatomía interna, su biología y su ecología. Existen varias publicaciones sobre la taxonomía de la familia, pero en realidad es poco lo que se discute y analiza de las especies del continente americano (Baker 1941, Binney 1879, Baker 1928, Thompson \& Lee 1980, Van Mol \& Van Bruggen 1971, Schileyko 2002).

Asimismo, las especies neotropicales de la familia Euconulidae tiene características llamativas e importantes que ameritan estudios muy detallados en campos tan diversos como morfología, anatomía, fisiología, etología, ecología y evolución. Por ejemplo, muchas de las especies de euconúlidos en Costa Rica presentan patrones de coloración del manto y no de la concha (como en otros grupos), además algunas especies tienen colores de manto inusuales entre los moluscos terrestres: azul, celeste y verde agua. Otro ejemplo es la musculatura del pie que permite a algunas especies hacer movimientos violentos (Binney 1879, Barrientos 1998). La mayor parte de los géneros de esta familia tienen conchas delgadas y traslúcidas, pero en 
algunos casos como en Velifera la concha llega a ser muy frágil o flexible (Shileyko 2002). El grupo presenta un cuerno caudal y una glándula de mucus con funciones desconocidas. Aunque en la literatura solo está reportada una especie de molusco terrestre bioluminiscente (Counsilman et al. 1987), en Costa Rica hay algunos casos nuevos pertenecientes a esta familia que deben ser estudiados y registrados.

\section{Formación de especies en moluscos}

La formación de especies es un proceso que puede darse en simpatría (Gittenberger 1988), alopatría (Buje 2007) o parapatría (Goodacre 2001). Hay parapatría cuando las poblaciones mantienen un flujo genético restringido que produce híbridos poco viables y refuerza la diferenciación prereproductiva de las especies (Tiller 1981). En la actualidad se reconocen cuatro mecanismos de especiación que se pueden dar tanto en simpatría como en alopatría o parapatría: ecológica, por divergencia bajo selección uniforme, por deriva genética y por reorganización genética (Pfenninger et al. 2003). En Costa Rica no se ha estudiado cuales de estos mecanismos se aplican a los diversos géneros, pero sin duda los actuales fenómenos de deforestación y cambio climático están incentivando tanto la especiación como la extinción de especies. Tal vez los primeros estudios en este campo podrían realizarse en las especies que habitan los páramos, ya que, la deriva genética podría ser un elemento importante en la formación de especies en esas zonas. En los análisis de especiación de los moluscos terrestres hay que tener presente que la dispersión en moluscos es lenta (algunos se desplazan menos de $5 \mathrm{~m}$ en toda su vida) (Pfenninger et al. 2003). En Costa Rica no se ha estudiado el desplazamiento de las especies, pero observaciones personales indican que el desplazamiento puede variar desde varios cientos de metros (por ejemplo en Euglandina spp. y Leptarionta spp.), hasta unos pocos centímetros o metros en el caso de especies de hojarasca (por ejemplo Charopidae, Helicodiscidae, Diplommatinidae). Esporádicamente los moluscos terrestres se desplazan largas distancias por medios pasivos (Tiller 1981, Goodacre 2001, Made \& Bellido 2007, Pfenninger et al. 2007). También se ha observado a varias especies de Subulinidae y Orthalicidae que fueron desplazadas largas distancias por crecidas de ríos, aunque los especímenes observados murieron ahogados, es posible que otros sobrevivieran (obser. pers.). No se debe dejar de lado el transporte pasivo de especies por medios modernos: maquinaria agrícola, automóviles, zapatos, etc. Para sobrevivir en nuevos hábitats requieren condiciones específicas, particularmente temperatura, humedad, acidez del suelo, hojarasca y compactación del suelo, condiciones que pueden afectar la morfología de las conchas (Tiller 1981, Barrientos 2000, Pfenninger et al. 2003, Pérez et al. 2006, 2008). Estas condiciones permiten el desarrollo de polimorfismos genéticos en las poblaciones y a que se mantengan debido a una baja tasa de intercambio (Chiba \& Davison 2007, Pfenninger et al. 2003, 2007). En Costa Rica puede ser que esos polimorfismos genéticos se presenten en varios géneros, como podrían ser Velifera, Habroconus y Euconulus. Para entender cuál es el proceso evolutivo que tuvo lugar en cada especie es necesario conocer su relación filogenética, distribución geográfica, historia natural (Pfenninger et al. 2003) y los procesos geológico-geográficos históricos (vulcanismo, sedimentación, glaciaciones, cambios en los cursos de los ríos, etc.) de la zona en estudio (Bunje 2007).

Considerando estas características de los moluscos, Pfenninger et al. (2003) propusieron un proceso de especiación en el que la especie: 1) expande su distribución en diferentes ecotonos, 2) se adapta a los ecotonos en forma de clinas, 3) un cambio ambiental elimina las variaciones intermedias en la clina dejando solo los extremos, 4) cuando las poblaciones remanentes vuelven a expandir su territorio ya han cambiado tanto que son especies distintas. Actualmente este patrón se presenta con frecuencia producto de la deforestación y la reforestación que ha experimentado Costa Rica. Aunque el esquema teórico este correcto puede ser un poco simplista, especialmente 
para moluscos terrestres. En este grupo los procesos evolutivos son más complejos debido a que el desplazamiento de las poblaciones es multidireccional (compárese con los moluscos dulceacuícolas que tienen un desplazamiento unidireccional principalmente) (Bunje 2007). Además, el territorio que ocupan puede expandirse y contraerse en múltiples ocasiones a través de la historia evolutiva de la especie (Pfenninger et al.2007) y pueden quedar aisladas subpoblaciones que se volverán a juntar si se vuelve a ampliar el territorio, o bien puede haber acontecimientos migratorios repetitivos de dispersión pasiva a larga distancia (Goodacre 2001, Hausdorf 2000). Un caso que sería muy interesante de estudiar es el de las especies que habitan en los páramos de Costa Rica, pues, estos ecosistemas se han expandido y unido en unas ocasiones, en otras se han contraído y aislado.

Las poblaciones de moluscos son unidades que evolucionan independientemente y se adaptan a las situaciones locales (Pfenninger et al. 2007). Dentro del modelo del proceso de especiación propuesto por Pfenninger et al. (2003), la formación de especies se puede dar en dos vías. La primera cuando las especies se diferencian primero a nivel genético y posteriormente, en simpatría, desarrollan diferencias morfológicas debido a competencia por alimento, reconocimiento de especies en la reproducción (Tiller 1981) y otras características ecológicas (Peterson et al. 1999). Por eso con frecuencia se encuentran linajes genéticamente distintos, pero ecológica y morfológicamente indistinguibles (Tiller 1981, Emberton 1995, Davison \& Chiba 2006, Bunje 2007). En estos casos la biogeografía histórica es más importante que el ambiente (Goodacre 2001, Made \& Bellido 2007). Aunque, es posible que en Costa Rica varios grupos presenten esta vía de especiación, no se han hecho estudios genéticos que apoyen la idea. La segunda vía se da cuando las especies se diferencian primero morfológicamente por adaptación ecológica y posteriormente se establecen las diferencias genéticas (Davison \& Chiba 2006). En Costa Rica géneros como Beckianum, Helicina, Velifera, Econulus, Habroconus, Euglandina, Spiraxis, entre otros presenten esta vía de formación de especies. Ambas vías pueden ser afectadas por las variaciones climáticas, especialmente por los cambios de humedad, ya que, estos afectan la morfología (Chiba \& Davison 2007, Tiller 1981, Pfenninger et al. 2007). Actualmente se esta desarrollando un estudio sobre los factores que afectan las diferencias morfológicas de Gastrocopta geminidens que habitan en Costa Rica, Nicaragua y Venezuela (López \& Barrientos en prep.). Igual efecto pueden tener los factores bióticos, tales como competidores y depredadores (Schilthuizen et al. 2006, Davison \& Chiba 2006, Chiba \& Davison 2007). En Costa Rica podría estudiarse el efecto de las planarias como depredadores de Urocoptidae en Palo Verde, Guanacaste.

Para poder distinguir claramente entre especies hermanas de moluscos, se necesita que haya pasado un tiempo considerable (entre 200000 y 3000000 de años) para que los diferentes linajes se diferencien de una manera marcada, de lo contrario se encontrarán poblaciones genéticamente muy homogéneas y que pueden hibridarse (Goodacre 2001, Bunje 2007). Aunque, el Istmo Centroamericano se terminó de formar hace 3000000 de años, varias partes del país tienen entre 5000000 y 70000000 de años, por lo que ha habido tiempo suficiente para que se formen distintas especies de moluscos terrestres. En comparación con otros grupos, los moluscos terrestres tienen tasas de evolución bastante altas, pues, ronda el $10 \%$ por millón de años y la divergencia genética entre especies puede estar entre 2 y $30 \%$. Se debe considerar que el reloj molecular que se utilice para estos estudios de moluscos terrestres estilomatóforos debe estar calibrado con otras especies hermafroditas (Pfenninger et al. 2003, Davison 2006). Por el contrario, la adaptación local de una especie se puede dar en un tiempo evolutivo corto, a veces en tan solo 100 años, ya sea por plasticidad fenotípica o por una innovación evolutiva o ambos (Peterson et al. 1999, Chiba \& Davison 2007, Pfenninger et al. 2003, 2007). La deforestación grave en Costa Rica tiene cerca de 100 años, lo que indica que es probable que varios grupos se 
hayan adaptado a las islas de vegetación que se formaron con la fragmentación de los bosques.

\section{Filogenia}

La filogenia de los moluscos neotropicales no ha sido estudiada. Generalmente, los estudios sobre este tema, aunque incluyen familias que están presentes en el neotrópico, utilizan especies de otras regiones. Un ejemplo es el caso de la familia Euconulidae, para la que solo se ha utilizado a Euconulus fulvus (una especie holártica) como representante de la familia, por lo que no se sabe si es monofilética o polifilética. Además, a nivel de familias, hay grandes variaciones entre los estudios (Emberton et al. 1990); por ejemplo Wade et al. (2001) consideran a los Euconulidae estrechamente relacionados con la familia Helicarionidae la cual consideran polifilética. Por su parte Hausdorf (1998) ubica a los Euconulidae cerca de Trochomorphidae. Barker (2001) los ubica cercanos a las familias: Dyakiidae, Agriolimacidae, Limacidae, Milacidae y Vitrinidae. Esas discordancias muestran la falta de datos y procedimientos genéticos robustos a nivel mundial, lo cual incluye los grandes vacíos de conocimiento de las especies tropicales.

\section{Distribución}

Es muy poco lo que se ha trabajado con la biogeografía de moluscos terrestres tropicales (Naranjo-García 2003) y no hay estudios al respecto de la fauna costarricense. La mayor parte de los moluscos terrestres tienen un ámbito de distribución de $50 \mathrm{~km}$ lineales y por lo general se pueden encontrar en simpatría entre cinco y 10 especies, rara vez superan las 20 especies (Solem 1984). Sin embargo, en algunos lugares tropicales se han encontrado cantidades sorprendentes en simpatría: 72 especies en Nueva Zelandia (Solem 1984) y 91 especies en Jamaica (Rosenberg \& Muratov 2006). Por lo general, en Costa Rica se encuentran entre 10 y 20 especies en un mismo sitio, excepto en lugares como el Valle del Silencio (Cordillera de Talamanca), en donde se han encontrado 40 especies (Barrientos 2003b), otro sitio con una gran cantidad de especies es Fila de Cal cerca de Ciudad Neilly. Para moluscos terrestres de Australasia se ha encontrado que el modelo de biogeografía de islas explica la presencia de las especies (Cameron et al. 2005). No obstante, para el análisis de distribución de las especies continentales debe tenerse en cuenta: la existencia de especiación simpátrica que puede ser frecuente en este grupo (Gittenberger 1988); la capacidad de las especies de dispersarse y establecerse (Hovenstadt \& Poethke 2005, Cowie \& Holland 2006, Pfenninger et al. 2007) y que el ámbito de distribución de una especie no es estático, se puede expandir y contraer múltiples veces. Además, se debe considerar los factores abióticos que determinan un microclima adecuado para los moluscos: un suelo geológico específico, topografía, temperatura, humedad, grosor y complejidad de la capa de hojarasca, compactación del suelo y diversidad de las comunidades vegetales (Solem 1984, Pfenninger et al. 2007, Pérez et al. 2006, 2008). De todos estos factores en Costa Rica solo se ha estudiado el efecto de la hojarasca (Barrientos 2000).

En el neotrópico los estudios de distribución realizados se refieren principalmente a la diversidad de algunas regiones; se desconocen todos los demás aspectos y no se han hecho estudios interdisciplinarios. Por ejemplo, Rangel-Ruíz et al. (2004) informaron 22 especies de moluscos terrestres para una región de Tabasco, México, y los principales componentes zoogeográficos fueron las especies endémicas y las de origen neotropical. Otros trabajos sólo informan la cantidad y lista de especies de moluscos terrestres en un país: 79 en el sur de Nuevo León, México (Correa-Sandoval et al. 2007), 104 en la vertiente pacífica de Nicaragua (Pérez \& López 2003), 159 en el noreste de México (Correa-Sandoval 2003), 183 en Costa Rica (Barrientos 2003a), 590 especies en Brasil (Campos-Salgado \& dos Santos-Coehlo 2003), 763 en Perú (Ramírez et al. 2003) y 1239 especies para México y Centroamérica (Thompson 2008). 


\section{Conservación}

Desde sus inicios la civilización humana ha inducido en el ambiente cambios que afectan la biodiversidad, esto incluye la de los moluscos terrestres (Preece 1998). Desde el punto de vista biológico, las variaciones ambientales llevan a sucesos de expansión, dispersión, invasión, migración, extinción, surgimiento de nuevas especies y alteraciones de los ámbitos de distribución de las especies (Monge-Nájera 2006), alterando y marcando los patrones biogeográficos naturales de las especies. En la actualidad las variaciones más dramáticas las producen la expansión de las fronteras agrícola y urbana y el cambio climático mundial. La deforestación asociada a la expansión de las actividades humanas ha producido espacios abiertos, con ámbitos de temperaturas y humedad mas amplios (Nebel 1999, Smith \& Smith 2001), lo que favorece a unas especies (en Costa Rica probablemente favorezca a subulínidos y a habitantes el bosque tropical seco) y desfavorecen a otras (en Costa Rica las especies que probablemente se desfavorezcan más son las que viven en bosques bien constituidos tales como los géneros Leptarionta, Labyrinthus y Cryptostrakon entre otras) (Lundholm 2006, Clergeau et al. 2006, Shochat et al. 2006). Los moluscos terrestres son un grupo muy susceptible a variaciones en la humedad por lo que también son afectados por la deforestación (Hyman 1967, South 1992, Cook 2001).

Algunos casos de especies de moluscos terrestres han expandido su hábitat, como Subulina octona, Zonitoides arboreus, Succinea costaricana y Helix aspersa (observ. pers.), están más asociadas con intervenciones humanas que con cambio climático. En la mayoría de los casos el cambio climático y de uso de la tierra está provocando que las especies se extingan (Barrientos 2003b, Lydeard et al. 2004). En el "libro rojo" de la UICN del 2002, el $42 \%$ de las extinciones de animales registradas pertenece a moluscos, de ese porcentaje el $99 \%$ eran especies continentales (dulceacuícolas y terrestres) (Lydeard et al. 2004). De Costa Rica se ha reportado la extinción de al menos 12 especies descritas y dos especies no descritas (Barrientos 2003b), lo que equivale al 7\% de las especies reportadas del país, sin embargo, estudios más detallados podrían revelar que la cifra es mayor. La destrucción del hábitat por expansión agrícola y urbana provoca también reemplazo de plantas nativas (que brindan las condiciones necesarias para que los moluscos sobrevivan) e introducción de depredadores (en Costa Rica se han introducido Rattus norvegicus, Mus musculus y Quiscalus mexicanus que muy probablemente tienen en su dieta moluscos terrestres) (Barrientos \& MongeNájera 2010a). Esas introducciones afectan a las poblaciones de moluscos de una forma significativa (Lydeard et al. 2004, Ohbayashi et al. 2007). Además, en el caso de los moluscos que suelen tener una baja capacidad de dispersión, la fragmentación del hábitat por causa humana, provoca que el flujo genético sea mas restringido (Pfenninger et al. 2007), por lo que muchas especies podrían estar en peligro de extinción y muchas se extinguirán antes de ser descritas (Roth 2003).

Los moluscos muestran afinidad con diferentes microhábitats. Algunos autores han destacado el aporte de las actuales técnicas agrícolas silvopastoriles, de agricultura orgánica y de restauración ecológica (Barrientos \& Monge-Nájera 2010b), en las que se evitan los monocultivos, se promueve el uso de especies nativas, se protege los reductos de bosque y se reduce el uso de plaguicidas. En la actualidad, estas técnicas han tenido un gran auge en Costa Rica y podrían estar favoreciendo especies nativas de moluscos gracias a una disminución de la expansión de especies introducidas e invasoras (Donald \& Evans 2006) y al mantenimiento de usos de suelo más compatibles con los requerimientos de los moluscos (Pérez et al. 2006). Dentro de los sistemas antes mencionados los moluscos son más diversos donde hay bosques primarios y tacotales (pastizales abandonados en los que han crecido una cantidad considerable de arbustos y otras plantas silvestres), que en pasturas con diferentes densidades de árboles, bosques secundarios con diferentes grados de intervención, bosques ribereños y cercas 
vivas (Pérez et al. 2006). Sin embargo, cuando la región está muy antropogenizada, como es la mayor parte del territorio costarricense que ha sido dedicado a la agricultura y ganadería, la diversidad de moluscos es mayor en las cercas vivas y en hábitats con iluminación filtrada y que tienen suelo húmedo suelto y con hojarasca diversa (Pérez et al. 2008); posiblemente esta preferencia se debe únicamente a la ausencia de otros hábitats más favorables. En el caso de zonas urbanas se ha encontrado que la protección de reservorios de agua que las surten pueden conservar a la mayor parte de los moluscos, especialmente si el $\mathrm{pH}$ del suelo es cercano a 7.3 y el hábitat incluye rocas (Clements et al. 2006). En Costa Rica la Ley 7575 artículo 33 protege todos los márgenes de ríos, manantiales y pozos, aunque no se han hecho estudios es probable que estos reservorios sean los únicos sitios que mantengan la malacofauna original de la Gran Área Metropolitana y cabeceras de provincia en Costa Rica.

A la hora de analizar las estrategias para la conservación de la biodiversidad se debe considerar que para que una población tenga $90 \%$ de probabilidad de mantenerse en los próximos 100 años, es necesario que al menos conste de 1377 individuos (Brook et al. 2006). También se debe considerar que la permanencia de las especies depende más de las características del hábitat que de sus propias características (Brook et al. 2006). De ahí la importancia de conocer el tamaño de las poblaciones de moluscos, información que está ausente para todas las especies de moluscos de Costa Rica, con excepción de Succinea costaricana en condiciones de plaga agrícola (Villalobos et al. 1995). Así mismo, en Costa Rica existen zonas de gran endemismo de moluscos terrestres, principalmente en las cadenas montañosas arriba de los 1 500msnm (Barrientos 2003b, 2005). En Nicaragua se reporta un endemismo del 17\% (Pérez et al. 2006), en tanto que Costa Rica, posee un endemismo de $30 \%$ para todo el país, aunque los datos en los que se basa ese cálculo fueron obtenidos principalmente en zonas a menos de $1500 \mathrm{msnm}$, que actualmente son las más urbanizadas y que no sobresalen por su endemismo (Barrientos 2003a,b, 2005). El cambio climático y de uso de la tierra puede tener grandes repercusiones en la malacofauna endémica y en las poblaciones de los moluscos terrestres, no obstante no se han realizado estudios que permitan determinar su estado de conservación.

La determinación de las zonas que deben conservarse se basa en gran medida, aunque no exclusivamente, en la identificación de "hotspots", es decir zonas con diversidad alta. Sin embargo, la escogencia de "hotspots" se basa, generalmente, en información de plantas, mamíferos y aves. Un estudio reciente realizado en el bosque tropical australiano demostró que los moluscos e insectos son buenos predictores de "hotspots" de plantas y vertebrados, pero no a la inversa (Lydeard et al. 2004). Por ello un estudio que identifique los "hotspots" de invertebrados podría dar información importante para la toma de decisiones más acertadas en cuanto a los lugares que deben ser protegidos. En Costa Rica para moluscos continentales es posible que los "hot spots" sean el Valle del Silencio y Fila de Cal, ambos en la Cordillera de Talamanca, pero se requiere de más estudios para determinarlos con exactitud. Para la conservación de los moluscos terrestres se ha recomendado: encontrar los "hotspots" de moluscos; generar datos históricos de distribución y abundancia; determinar la ecología de las especies y del papel de estas en el ecosistema para determinar su vulnerabilidad; conservar principalmente las riberas de los ríos y cercas vivas cuando el terreno está muy antropogenizado; e identificar especies y linajes con historia evolutiva independiente con el fin de conservar la biodiversidad genética (Taylor et al. 1994, Lydeard et al. 2004, Barrientos 2005, Pérez et al. 2006, Backeljau et al. 2001).

Costa Rica es ampliamente conocida por su gran biodiversidad, esto por supuesto incluye también a grupos de invertebrados como los moluscos terrestres, grupo que además presenta un alto nivel de endemismos. El conocimiento que existe de los moluscos costarricenses es mínimo en todos los campos. Para lograr una adecuada conservación de este grupo es 
importante conocer con mayor exactitud cuáles son las especies que están en el país y su diversidad morfológica y genética, así como sus afinidades taxonómicas. La conservación de este grupo no puede darse si no se conocen los requerimientos de nicho de las distintas especies, su distribución y abundancia. También es importante determinar cuáles factores bióticos y abióticos están afectando a las poblaciones y aunque el cambio del uso de suelo en Costa Rica esta prohibido, la realidad es que se siguen ampliando las zonas urbanas y se desconoce cual es el impacto que esto tiene sobre las poblaciones de moluscos. Es importante determinar cuales son los centros de alta diversidad de moluscos y corroborar que se les este dando protección de alguna manera.

\section{AGRADECIMIENTOS}

Se agradece el trabajo asistencial de Maribel Zúñiga y las revisiones de Edna Naranjo, Edmund Gittenberger y Julián Monge. Este trabajo es parte del trabajo doctoral de la autora dentro del Doctorado en Ciencias Naturales para el Desarrollo (DOCINADE) programa conjunto de la Universidad Nacional (UNA), Costa Rica, Instituto Tecnológico de Costa Rica (TEC) y la Universidad Estatal a Distancia (UNED), Costa Rica. La autora esta matriculada en la UNED.

\section{RESUMEN}

Los moluscos terrestres son un grupo muy poco estudiado a nivel mundial. Hay 183 especies reportadas para Costa Rica, $30 \%$ son endémicas y $7 \%$ posiblemente están extintas. Se espera que en el país haya alrededor de 400 especies, de más del 95\% se desconoce su biología, ecología, distribución, genética y otros campos de estudio. En Costa Rica las familias con mayor número de especies son Spiraxidae, Orthalicidae y Subulinidae. No obstante, es posible que Euconulidae sea aun más diversa, pues habitan las zonas altas del país que es en donde menos trabajo se ha hecho. El estudio de regiones altas aumentará el porcentaje de endemismo. Los futuros trabajos malacológicos taxonómicos, biológicos y ecológicos deben considerar la poca movilidad de este grupo, su tendencia a formar especies nuevas en simpatría, la especificidad de requerimientos de microhábitat, el hermafroditismo, la alta tasa de evolución
(10\% por millón de años) y la divergencia entre especies (2-30\%). Para proteger adecuadamente la biodiversidad de la malacofauna costarricense, se requiere de estudios que determinen la distribución y abundancia de las especies y el efecto del uso de la tierra y del climático.

Palabras clave: moluscos terrestres, endemismo, conservación de invertebrados.

\section{REFERENCIAS}

Achuba, F. 2008. African land snail Achatina marginatus, as bioindicator of environmental pollution. NorthWest J. Zoo. 4: 1-5.

Andrews, K., V.H. Valverde \& O. Ramírez.1985. Preferencia alimenticia de la babosa, Sarasinula plebeia (Fisher). Ceiba 26: 59-65.

Backeljau, T., A. Baur \& B. Baur. 2001. Population and conservation genetics, p. 383-412. In G.M. Baker (ed.). The biology of terrestrial mollusks. CABI, Nueva York, EEUU.

Baker, H.B. 1928. Minute American Zonitidae. Proc. Acad. Nat. Sc. Phil. 80: 1-44.

Baker, H.B. 1941. Zonitid snails from pacific islands. Parts 3 and 4. 3. Genera other than microcystinae 4. Distribution and indexes. Bernice P. Bishop Museum, Honolulu, Hawaii, EEUU.

Barker, G.M. 2001. Gastropods on Land: Phylogeny, Diversity and Adaptative Morphology, p. 1-146. In G.M. Barker (ed.). The biology of terrestrial mollusks. CABI. Nueva York, EEUU.

Barrientos, Z. 1998. Life history of the terrestrial snail Ovachlamys fulgens (Stylommatophora: Helicarionidae) under laboratory conditions. Rev. Biol. Trop. 46: 369-384.

Barrientos, Z. 2000. Populations dynamics and spatial distribution of the terrestrial snail Ovachlamys fulgens (Stylommatophora: Helicarionidae) in a tropical environment. Rev. Biol. Trop. 48: 71-87.

Barrientos, Z. 2003a. Lista de especies de moluscos terrestres (Archaeogastropoda, Mesogastropoda, Archaeopulmnata, Stylommatophora, Soleolifera) informadas para Costa Rica. Rev. Biol. Trop. 51: 293-304.

Barrientos, Z. 2003b. Estado actual del conocimiento y la conservación de los moluscos continentales de Costa Rica. Rev. Biol. Trop. 51: 285-292. 
Barrientos, Z. 2005. Moluscos terrestres de los páramos de Costa Rica, p. 501-509. In M. Kapelle \& S.P. Horn (eds.). Páramos de Costa Rica. Editorial INBio, Heredia, Costa Rica.

Barrientos, Z. \& J. Monge-Nájera. 2010a. Especies introducidas en la Meseta Central de Costa Rica. Biocenosis (en prensa).

Barrientos, Z. \& J. Monge-Nájera. 2010b. Restauración ecológica en la Meseta Central de Costa Rica. Biocenosis (en prensa).

Binney, W.G. 1879. On the jaw and lingual dentition of certain Costa Rica Land Shells collected by Dr. William M. Gabb. Ann. N.Y. Acad. Sci. 1: 257-262.

Brook, B.W., L.W. Traill \& C.J. Bradshaw. 2006. Minimum viable population sizes and global extinction risk are unrelated. Ecol. Lett. 9: 375-382.

Bunje, P.M.E. 2007. Fluvial range expansion, allopatry, and parallel evolution in a Danubian snail lineage (Neritidae: Theodoxus). Biol. J. Linn. Soc. 90: 603-617.

Cameron, R.A., B.M. Pokryszko \& F.E. Wells. 2005. Alan Solem's work on the diversity of Australasian land snails: an unfinished project of global significance. Rec. West. Aus. Mus.: 1-10.

Campos-Salgado, N. \& A.C. Dos Santos-Coelho. 2003. Moluscos terrestres do Brasil (Gastropodes operculados ou não, exclusive Veronicellidae, Milacidae e Limacidae). Rev. Biol. Trop. 51: 149-189.

Chiba, S. \& A. Davison. 2007. Shell shape and habitat use in the north-west Pacific land snail Mandarina polita from Hahajima, Ogasawara: current adaptation or ghost of species past? Biol. J. Linn. Soc. 91: 149-159.

Clements, R., L. Koh, T. Lee, R. Meier \& D. Li. 2006. Importance of reservoirs for the conservation of freshwater molluscs in a tropical urban landscape. Biol. Conserv. 136-146.

Clergeau, P., J. Jokimäki \& R. Snep. 2006. Using hierarchical levels for urban ecology. Trends Ecol. Evol. 21: $660-661$

Cook, A. 2001. Behavioural ecology: on doing the right thing, in the right place at the right time, p. 447488. In G.M. Barker (ed.). The biology of terrestrial mollusks. CABI, Nueva York, EEUU.

Correa-Sandoval, A. 2003. Gastrópodos terrestres del Noreste de México. Rev. Biol. Trop. 51: 507-522.
Correa-Sandoval, A., N.E. Strenth \& M.C. Salazar-Rodríguez. 2007. Zoogeografía de los gasterópodos terrestres del Sur de Nuevo León México. Acta Zool. Mex. 23: 143-162 (También disponible en línea: http:// redalyc.uaemex.mx/redalyc/pdf/575/57523208.pdf).

Cowie, R.H. \& B.S. Holland. 2006. Dispersal is fundamental to biogeography and the evolution of biodiversity on oceanic islands. J. Biogeogr. 33: 193-198 (También disponible en línea: http://www.biomas.ufop.br/ biomas/biog/roberthWill.pdf).

Counsilman, J.J., D. Loh, S.Y. Chan, W.H. Tan, J. Copeland \& M. Maneri. 1987. Factors affecting the rate of flashing and loss of luminescence in an Asian land snail, Dyakia striata. The Veliger 29: 394-399.

Dallinger, R., B. Berger, R. Triebskorn-Köhler. 2001. Soil biology and ecotoxicology, p. 489-525. In G.M. Barker (ed.). The biology of terrestrial mollusks. CABI, Nueva York, EEUU.

Davison, A. 2006. The ovotestis: an underdeveloped organ of evolution. BioEssays 28: 642-650.

Davison, A. \& S. Chiba. 2006. Labile ecotypes accompany rapid cladogenesis in an adaptative radiation of Mandarina (Bradybaenidae) land snails. Biol. J. Linn. Soc. 88: 269-282.

Donald, P.F. \& A.D. Evans. 2006. Habitat connectivity and matrix restoration: the wider implications of agrienvironment schemes. J. Appl. Ecol. 43: 209-218.

Emberton, K.C. 1995. Sympatric convergence and environmental correlation between two land-snails species. Evol. 49: 469-475.

Emberton, K.C., G.S. Kuncio, G.M. Davis, S.M. Phillips, K.M. Monderewicz \& Y.H. Guo. 1990. Comparison of recent classifications of Stylommatophoran landsnail families, and evaluation of large-ribosomalRNA sequencing for their phylogenetics. Malacologia 31: $327-352$.

Gittenberger, E. 1988. Sympatric speciation in snails: a largely neglected model. Evol. 42: 826-828.

Goodacre, S.L. 2001. Genetic variation in a Pacific Island land snail: population history versus current drift and selection. Proc. R. Soc. Lond. B. 268: 121-126.

Hausdorf, B. 1998. Phylogeny of the Limacoidea sensus lato (Gastropoda: Stylommatophora). J. Moll. Stud. 64: $35-66$.

Hausdorf, B. 2000. Biogeography of the Limacoidea sensus lato (Gastropoda: Stylommatophora): vicariance 
events and long-distance dispersal. J. Biogeogr. 27: 379-390.

Hovenstadt, T. \& H.J. Poethke. 2005. Dispersal and establishment: spatial patterns and species-area relationships. Divers. Distrib. 11: 333-340.

Hyman, L.H. 1967. The invertebrates: Mollusca 1(Vol VI). McGraw-Hill, Nueva York, EEUU.

Jennings, T.J. \& Barkham, J.P. 1979. Litter decomposition by slugs in mixed deciduous woodland. Holarctic Ecol. 2: 21-29.

Lundholm, J.T. 2006. How novel are urban ecosystems? Trends Ecol. Evol. 21: 659-660.

Lydeard, C., R.W. Cowie, W.F. Ponder, A.E. Bogan, P. Bouchet, S.A. Clark, K.S. Cummings, T.J. Frest, O. Gargominy, D.G. Herbert, R. Hersheler, K.E. Perez, B. Roth, M. Seddon, E.E. Strong \& F.G. Thompson. 2004. The Global decline of nonmarine mollusks. BioScience 54: 321-330.

Made, L. \& A. Bellido. 2007. Spatial variation of shell morphometrics in the subantarctic land snail Notodiscus hookeri from Crozet and Kerguelen Islands. Polar Biol. 30: 1571-1578.

Margulis, L. \& K.V. Schwartz. 1998. Five Kingdoms: An Illustrated Guide to the Phyla of Life on Earth. Freeman, Nueva York, EEUU.

Monge-Nájera, J. 1997. Moluscos de importancia agrícola y sanitaria en el trópico: la experienca costarricense. Universidad de Costa Rica, San José, Costa Rica.

Monge-Nájera, J. 2006. Ecological biogeography: a review with emphasis on conservation and the neutral model. Gayana 72: 102-112.

Naranjo-García, E. 2003. Moluscos continentales de México: Terrestres. Rev. Biol. Trop. 51: 483-493.

Nebel, B.J. 1999. Ciencias Ambientales. Ecología y desarrollo sostenible. Prentice Hall, México D.F., México.

Ohbayashi, T., I. Okochi, H. Sato, T. Ono \& S. Chiba. 2007. Rapid decline of endemic snails in the Ogasawara Islands, Western Pacific Ocean. Appl. Entomol. Zool. 42: 479-485.

Pérez, A.M. \& A. López. 2003. Listado de la malacofauna continental (Mollusca: Gastropoda) del Pacífico de Nicaragua. Rev. Biol. Trop. 51: 405-451.

Pérez, A.M., M. Sotelo, F. Ramírez, I. Ramírez, A. López \& I. Síria. 2006. Conservación de la biodiversidad en sistemas agropastoriles de Matiguás y Río Blanco (Matagalpa, Nicaragua). Ecosistemas 15: 125-14 (también disponible en línea: http://www.revistaecosistemas.net/pdfs/429.pdf).

Pérez, A.M., M. Sotelo, I. Arana \& A. López. 2008. Diversidad de moluscos gasterópodos terrestres en la región del Pacífico de Nicaragua y sus preferencias de hábitat. Rev. Biol. Trop. 56: 317-332.

Peterson, A.T., J. Soberón \& V. Sánchez-Cordero. 1999. Conservatism of Ecological niches in evolutionary time. Science 285: 1265-1267.

Pfenninger, M., A. Eppenstein \& F. Magnin. 2003. Evidence for ecological speciation in the sister species Candidula unifasciata (Poiret, 1801) and C. rugosiuscula (Michaud, 1831) (Helicellinae, Gastropoda). Biol. J. Linn. Soc. 79: 611-628.

Pfenninger, M., C. Nowak \& P. Magnin. 2007. Intraespecific range dynamics and niche evolution in Candidula land snail species. Biol. J. Linn. Soc. 90: 303-317.

Preece, R.C. 1998. Impact of early Polynesian occupation on the land snail fauna of Henderson Islands, Pitcairn group (South Pacific). Phil. Trans. R. Soc. Lond. B 353: 347-368.

Ramírez, R., C. Paredes \& J. Arenas. 2003. Moluscos del Perú. Rev. Biol. Trop. 51: 225-284.

Rangel-Ruíz, L.J., J. Gamboa-Aguilar \& F. Alegría-Ruíz. 2004. Diversidad malacológica de la región Maya. II. "Parque estatal Agua Blanca", Tabasco, México. Acta Zool. Mex. 20: 55-62 (también disponible en línea: http://redalyc.uaemex.mx/redalyc/pdf/575/57520105. pdf).

Rosenberg, G. \& I.V. Muratov. 2006. Status report on the terrestrial Mollusca of Jamaica. P. Acad. Nat. Sci. Phil. 155: 117-161.

Roth, B. 2003. Polygyrid land snails, Vespericola (Gastropoda: Pulmonata), 4. A new and possibly extinct species form central California, U.S.A. Zootaxa 215: 1-6.

Rousseau, D.D. 1992. Terrestrial mollusks as indicator of global Aeolian dust fluxes during glacial stages. Boreas 21: 105-109.

Sazima, I. 1989. Feeding behavior of the snail-eating snake Dipsas indica. J. Herpetol. 23: 464-468.

Schileyko, A.A. 2002. Treatise on recent terrestrial pulmonate mollusks: Punctidae, Helicodiscidae, Discidae, Cystopeltidae, Euconulidae, Trochomorphidae. Ruthenica 8: 1035-1166. 
Schilthuizen, M., A. Van Til, M. Salverda, T.S. Liew, S. James, B. Bin-Elahan \& J.J. Vermeulen. 2006. Microgeographic evolution of snail shell shape and predator behavior. Evol. 60: 1851-1858.

Shochat, E., P.S. Warren \& S.H. Faeth. 2006. Future directions in urban ecology. Trends Ecol. Evol. 21: 661-662.

Smith, R.L. \& T.M. Smith. 2001. Ecología. Pearson, Madrid, España.

Solem, A. 1984. A world model of land snail diversity and abundance, p. 6-22. In A. Solem \& A.C. Van Bruggen (eds.). World-wide snails: Biogeographical studies on non-marine Mollusca. E.J. Brill, Leiden, Holanda (también disponible en línea: http://books.google. co.cr/books)

South, A. 1992. Terrestrial Slugs: Biology, ecology and control. Chapman \& Hall, Londres, Inglaterra.

Taylor, R.J., R. Mesibov \& I. Growns. 1994. Local distribution patterns of land snails in relation to vegetation: Implications for reserve design. Mem. Queensland Mus. 36: 215-220

Thompson, F.G. \& H.G. Lee. 1980. A new helicarionid land snail from the southeastern United States. Malacological Rev. 13: 37-44 (también disponible en línea: http://www.flmnh.ufl.edu/malacology/mexicocentral_america_snail_checklist/).

Thompson, F.G. 2008. An annotated checklist and bibliography of the land and freshwater snails of Mexico and Central America. Florida Museum of Natural History, University of Florida, Florida, EEUU. (Consultado 23 marzo 2010, http://www.flmnh.ufl.edu/malacology/ mexico-central_america_snail_checklist/).

Tiller, S. 1981. Clines, convergence and character displacement in New Caledonian diplommatinids (land prosobranchs). Malacologia 21: 177-208.

Van Mol, J.J. \& A.C. Van Bruggen. 1971. Description d'un nouveau genre d'Euconulidae d'Afrique et discussion sur les affinités systématiques de cette famille (Mollusca, Gastropoda Pulmonata). Rev. Zool. Bot. Afr. 84: 285-296.

Villalobos M.C., J. Monge-Nájera, Z. Barrientos \& J. Franco. 1995. Life cycle and field abundance of the snail Succinea costaricana (Stylommatophora: Succineidae), a tropical agricultural pest. Rev. Biol. Trop. 43: 181-188.

Wade, C.M., P.B. Mordan \& B. Clarke. 2001. A phylogeny of the land snails (Gastropoda: Pulmonata). Proc. R. Soc. Lond. B 268: 413-422. 